\title{
Distribución latitudinal y batimétrica de las especies más abundantes y frecuentes en la fauna acompañante del camarón del Golfo de California, México
}

\author{
Carlos Hiram Rábago-Quiroz ${ }^{1,2}$, Juana López-Martínez ${ }^{2 *}$, José Eduardo Valdez-Holguín ${ }^{1}$ \& \\ Manuel O. Nevárez Martínez ${ }^{3}$ \\ 1. Departamento de Investigaciones Científicas y Tecnológicas de la Universidad de Sonora (DICTUS), Luis Donaldo \\ Colosio S/N, entre Sahuaripa y Reforma, Colonia Centro, C.P. 83000, Hermosillo, Sonora, México; \\ crabago_28@yahoo.com.mx, jvaldez@guayacan.uson.mx \\ 2. Centro de Investigaciones Biológicas del Noroeste, Unidad Sonora, Campus Guaymas, Km. 2.35 carretera a las \\ Tinajas, S/N Col. Tinajas, C.P. 85465, Guaymas, Sonora, México; jlopez04@ @ibnor.mx \\ 3. Centro Regional de Investigación Pesquera Guaymas. Calle 20 Sur No. 605, Colonia La Cantera, C.P. 45400, \\ Guaymas, Sonora, México; manuel.nevarez@prodigy.net.mx \\ * Correspondencia.
}

Recibido 22-III-2010. Corregido 25-IX-2010. Aceptado 28-X-2010.

\begin{abstract}
Latitudinal and bathymetric distribution of the most abundant and frequent species in the shrimp bycatch from the Gulf of California, Mexico. The Gulf of California is one of the most mega-diverse regions in the world, for which few fishery information is available. We present here latitudinal and bathymetric distribution of the most abundant and frequent bycatch species from the Gulf of California. The samples were obtained from a total of 111 hauls taken during seven research cruises of the closed shrimp season (2002-20052007), and also, from research cruises made at depths up to $90 \mathrm{~m}$. Due to the high variety species in this experimental shrimp bycatch, only those with highest biological value index (BVI) were selected. A total of fifteen species had the highest BVI and represented about $60 \%$ of the total abundance. A total of 16508 organisms were analyzed, representing 243 fish, crustacean, mollusk and echinoderm species. Fish were the most abundant, being the most frequent species: Urobatis halleri, Synodus scituliceps, Diplectrum pacificum, Haemulopsis nitidus and Eucinostomus argenteus. A wide latitudinal distribution of these species along the study area, as well as a bathymetric distribution from 9 to $67 \mathrm{~m}$ depth, was observed. Two of these species were found at $325 \mathrm{~m}$ depth. Due to the wide bathymetric distribution obtained, total abundances and sizes for each species by depth strata should be determined, and one can assume that deeper than $25 \mathrm{~m}$, the capture of these species decreases, and these areas can be used as natural repopulation areas, for depths where they are mainly captured by the commercial shrimp fishery. Rev. Biol. Trop. 59 (1): 255-267. Epub 2011 March 01.
\end{abstract}

Key word: biological value index, shrimp trawl, closed shrimp season, shrimp by-catch, fishes.

En el ámbito internacional una de las preocupaciones más significativas en el manejo y conservación de los ecosistemas marinos explotados, es la mortalidad de especies capturadas incidentalmente (Pope et al. 2000, Davis 2002). En este sentido, las pesquerías de crustáceos bentónicos con redes de arrastre, contribuyen en gran medida a esta problemática, fundamentalmente debido a la baja selectividad de las artes de pesca utilizadas en la captura del recurso objetivo. Según la FAO, la pesquería de camarón en zonas tropicales genera alrededor de 1.86 millones de toneladas de captura incidental representando $27.3 \%$ del total de la captura incidental de las pesquerías comerciales del mundo (Kelleher 2005).

La pesquería multiespecífica de camarón de altamar es una de las más importantes en el 
Golfo de California, siendo una fuente significativa de ingresos y empleo para las comunidades a lo largo del Golfo (López-Martínez et al. 2001); sin embargo, esta pesquería es también una de las que más contribuye a la problemática de las capturas incidentales en México, generando alrededor de 114000 toneladas de peces descartados al año, con una biomasa total estimada en $(90 \pm 45) \times 10^{3}$ toneladas (MadridVera et al. 2007).

Las redes de arrastre utilizadas por la flota camaronera en el Golfo de California capturan además del camarón (Farfantepenaeus californiensis Holmes, 1900, Litopenaeus stylirostris Stimpson, 1874, Litopenaeus vannamei Boone, 1931, entre otros), varias especies a las que se le conoce como fauna acompañante del camarón (FAC), siendo la mayoría peces bentónicos o epibentónicos (Pérez-Mellado \& Finley 1985). Aunque la magnitud real de los efectos sobre el ecosistema son desconocidos, el descarte de estos recursos representa un claro desperdicio de especies que pudieran ser aprovechadas como fuente de alimento (Bojorquez 1998, Rábago-Quiroz et al. 2008).

Los arrastres camaroneros se llevan a cabo en la zona costera entre los 9 y $90 \mathrm{~m}$ de profundidad, en regiones donde se concentra una gran variedad de especies (López-Martínez et al. 2007a) en las que existen fuertes presiones de grupos conservacionistas por prohibir este tipo de pesca. A la fecha se han planteado diversos mecanismos de conservación, tales como cierre de áreas a la pesca, restricciones de artes de pesca y la implementación de nuevas tecnologías (López-Martínez et al. 2007a). Sin embargo, la formulación de tales procedimientos requiere: identificar áreas y localidades donde hayan ocurrido reducciones en las capturas de esta fauna acompañante por unidad de esfuerzo, así como identificar cambios espaciotemporales en las abundancias de estas especies (Kanyerere 2001).

Son pocos los trabajos que se han realizado en el área sobre la distribución y abundancia de las especies que componen la fauna acompañante del camarón de altamar en el Golfo de California, información clave para dilucidar potenciales efectos de las artes de pesca sobre la composición de especies, distribución y su importancia numérica en el total de la fauna acompañante (Pope et al. 2000, Rueda \& Defeo 2001, Davis 2002, Stobutzki et al. 2003). Este trabajo tiene como objetivo determinar la distribución latitudinal y batimétrica de las especies más frecuentes y abundantes que componen la fauna acompañante del camarón en el Golfo de California, México.

\section{MATERIALES Y MÉTODOS}

Para el desarrollo de esta investigación se contó con dos fuentes de información:

1. Muestras de la fauna acompañante del camarón de lances obtenidos en cruceros realizados en época de veda de la pesquería del camarón (de marzo a agosto) en el Golfo de California, durante los años 2002 a 2005 y 2007 (Cuadro 1, Fig. 1A). Estos cruceros fueron realizados por el Instituto Nacional de Pesca (INAPESCA) durante los meses de julio y agosto, con una duración de dos semanas y siguiendo un derrotero sistemático de 60 estaciones, y están orientados a evaluar la distribución y abundancia de las especies de camarón que son capturadas comercialmente (Fig. 1A). Los arrastres se efectuaron durante una hora con redes de arrastre camaroneras, con una luz de malla de $50 \mathrm{~mm}, 30.5 \mathrm{~m}$ de abertura (boca) y una longitud de $30 \mathrm{~m}$. En general, el sistema de captura utilizado en estos cruceros es similar a aquél de la flota comercial, diferenciándose en que ésta opera en áreas de alta concentración de camarón, conocidas como "caladeros". Por lo que la información obtenida de estos muestreos no estaría tan sesgada en cuanto a muestrear solo estas áreas.

El material biológico que se obtuvo en los arrastres se vació en cubierta y se obtuvieron muestras de $20 \mathrm{~kg}$ (cantidad estimada por la FAO para estudios de fauna acompañante del camarón); los ejemplares 


\section{CUADRO 1}

Número de lances muestreados y número de organismos analizados para cada uno de los tipos de prospecciones realizadas de 2002 al 2005 y 2007 en el Golfo de California

TABLE 1

Number of shrimp trawls sampled and number of organisms analyzed for each research cruises in the Gulf of California during 2002-2005 and 2007

\begin{tabular}{lccl} 
Tipo/Fecha de Muestreos & $\begin{array}{c}\text { Num. de lances } \\
\text { muestreados }\end{array}$ & $\begin{array}{c}\text { Num. de organismos } \\
\text { analizados }\end{array}$ & \multicolumn{1}{c}{$\begin{array}{c}\text { Localidad } \\
\text { (Zona del Golfo) }\end{array}$} \\
Veda Agosto 2002 & 9 & 728 & Centro y Sur \\
Veda Julio 2003 & 6 & 799 & Sur del Golfo \\
Veda Julio 2004 & 16 & 5048 & Norte, Centro y Sur \\
Veda Agosto 2004 & 5 & 1307 & Centro y Sur \\
Veda Agosto 2005 & 10 & 2133 & Norte, Centro y Sur \\
Veda Julio 2007 & 58 & 5796 & Norte, Centro y Sur \\
Veda Agosto 2007 & 7 & 697 & Norte, Centro y Sur \\
Profundidad Sept 2004 & 11 & 1720 & Centro del Golfo \\
Profundidad Febrero 2005 & 29 & 3834 & Centro y Sur \\
Profundidad Mayo 2005 & 31 & 4566 & Norte, Centro y Sur \\
Profundidad Junio 2007 & 20 & 5097 & Norte, Centro y Sur \\
Profundidad Octubre 2007 & 25 & 3951 & Centro del Golfo
\end{tabular}

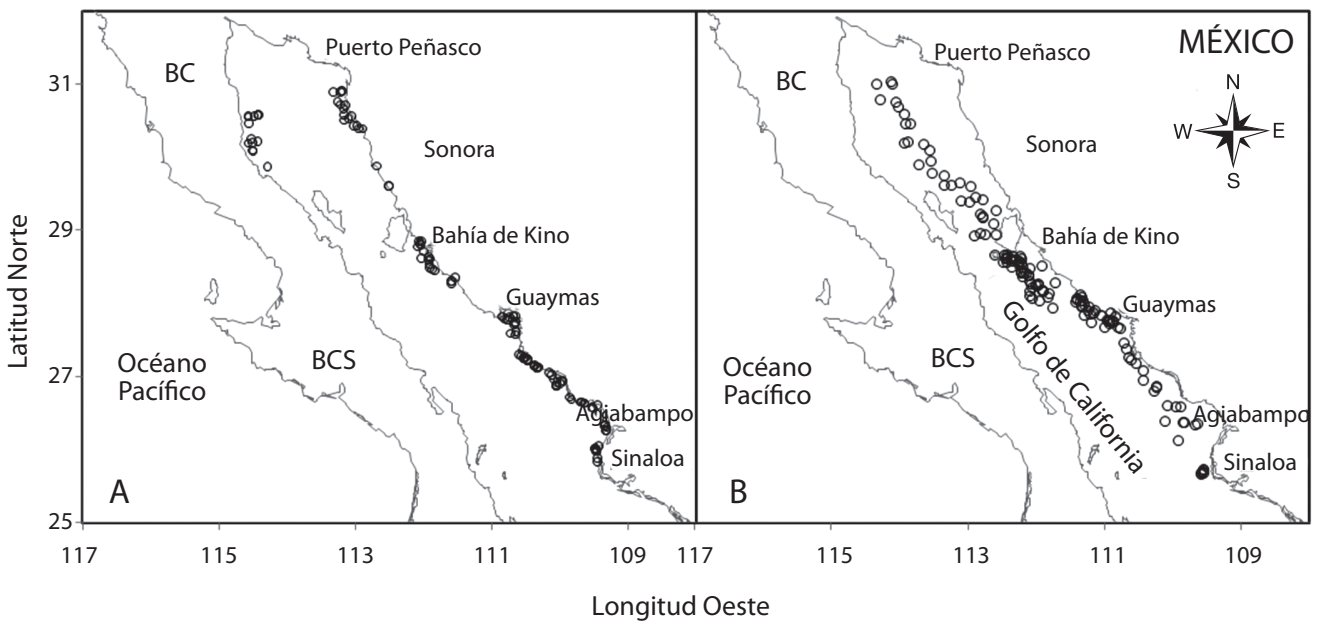

Fig. 1. Áreas cubiertas durante: (A) cruceros de veda del camarón de 2002 al 2005 y 2007; y (B) cruceros realizados a profundidades mayores a los 90m de 2004 al 2007 en el Golfo de California, México.

Fig. 1. Covered areas during: (A) research cruises in the closed shrimp season (2002-2005 and 2007); and (B) research cruises made in depth up 90m (2004-2007) in the Gulf of California, Mexico. 
recolectados se separaron por familia y género, y se congelaron hasta su procesamiento en laboratorio. La identificación y procesamiento del material biológico se realizó en los laboratorios de pesquerías e ictiología del Centro de Investigaciones Biológicas del Noroeste (CIBNOR, SC), sede en Guaymas, Sonora y La Paz, Baja California Sur (BCS).

La identificación taxonómica de las especies se realizó mediante las claves y descripciones de Jordan \& Evermann (1896-1900), Meek \& Hildebrand (1923-1928), Miller \& Lea (1976), Eschmeyer et al. (1983), Fischer et al. (1995), Robertson \& Allen (2002). Ejemplares de referencia fueron fijados con formaldehído a $10 \%$ y posteriormente preservados en alcohol etílico a $70 \%$. Este material se encuentra en el laboratorio de Investigación Pesquera del CIBNOR, SC, Unidad Guaymas, para posteriormente ser incorporado a la Colección Ictiológica del Centro. Toda esta información fue registrada, georeferenciada y concentrada en bases de datos para su análisis.

2. Debido a que posiblemente algunas de las especies más abundantes y frecuentes en la fauna acompañante del camarón tienen una distribución batimétrica más allá de la profundidad en la que opera la flota camaronera $(>90 \mathrm{~m})$, se obtuvo información de la fauna acompañante de lances provenientes de cinco cruceros prospectivos orientados a la evaluación del camarón localizado a profundidades mayores a los 90m efectuados por el CIBNOR, SC. Los cruceros fueron efectuados en el Golfo de California entre los años 2004 y 2007 (Cuadro 1, Fig. 1B). La red usada fue una de arrastre de fondo construida de polietileno, hilo trenzado de tamaño 30/31 con un perímetro de entrada de $60 \mathrm{~m}$ con un tren de arrastre de $7.6 \mathrm{~cm}$ de diámetro, operada por popa y con una luz de malla de $40 \mathrm{~mm}$, $45 \mathrm{~mm}$ y $50 \mathrm{~mm}$, en las distintas partes del cuerpo. El procesamiento de las muestras fue el mismo que para las provenientes de los cruceros en época de veda del camarón.

Para determinar las especies de mayor importancia biológica en la fauna acompañante del camarón, se utilizó el índice de valor biológico (IVB) descrito por Sanders (1960, citado por Loya-Salinas \& Escofet 1990). Debido al gran número de especies presentes en la fauna acompañante del camarón, se eligieron las 15 especies de mayor importancia biológica, las cuales obtuvieron los mayores puntajes en el IVB y que a la vez representaron más de $55 \%$ de la abundancia del total de las especies.

Se obtuvieron los gráficos de distribución latitudinal y batimétrica de estas 15 especies, combinando los dos tipos de información (de cruceros en época de veda y de prospecciones a profundidades mayores a los $90 \mathrm{~m}$ ) para así tener una mejor representación de las distribuciones de cada especie.

Tratando de identificar posibles asociaciones y la ordenación de las especies de la fauna acompañante del camarón con una determinada zona del Golfo de California y comparar estas con las 15 especies más abundantes y frecuentes obtenidas a través del IVB, se realizó un análisis de correspondencia a través del programa estadístico JMP.

\section{RESULTADOS}

Se analizaron 111 lances de pesca en los 7 cruceros de veda efectuados entre los años 2002 a 2005 y 2007. Se obtuvo un total de 16,508 organismos en las muestras, representando 243 especies entre peces, crustáceos, moluscos y equinodermos, siendo el grupo de los peces el más abundante con más de $95 \%$ de las capturas.

Las 15 especies con el IVB más alto en la fauna acompañante del camarón durante estos cruceros se presentan en la Cuadro 2. Todas ellas correspondieron a peces y representaron más de $58 \%$ de la abundancia total. Las especies más recurrentes fueron: Synodus 
CUADRO 2

Especies con el IVB más alto en la fauna de acompañamiento del camarón durante cruceros de veda del 2002 al 2005 y 2007 en el Golfo de California

TABLE 2

Species with the highest BVI in the shrimp bycatch during shrimp closed season cruises in the Gulf of California during 2002-2005 and 2007

\begin{tabular}{llcccc}
\multicolumn{1}{c}{ Especie } & \multicolumn{1}{c}{ Familia } & Abu (\# Org) & Abr \% & Ara \% & Pun (IVB) \\
Urobatis halleri & Urotrygonidae & 1469 & 8.89 & 8.89 & 411 \\
Synodus scituliceps & Synodontidae & 560 & 3.39 & 12.29 & 349 \\
Diplectrum pacificum & Serranidae & 542 & 3.28 & 15.57 & 306 \\
Haemulopsis nitidus & Haemulidae & 1091 & 6.61 & 22.17 & 303 \\
Eucinostomus argenteus & Gerreidae & 977 & 5.92 & 28.09 & 282 \\
Pomadasys panamensis & Haemulidae & 561 & 3.40 & 31.49 & 232 \\
Eucinostomus gracilis & Gerreidae & 589 & 3.57 & 35.05 & 228 \\
Eucinostomus entomelas & Gerreidae & 709 & 4.29 & 39.35 & 212 \\
Balistes polylepis & Balistidae & 321 & 1.94 & 41.29 & 163 \\
Paralabrax maculatofasciatus & Serranidae & 287 & 1.74 & 43.03 & 162 \\
Porichthys analis & Batrachoididae & 676 & 4.09 & 47.12 & 153 \\
Micropogonias megalops & Sciaenidae & 371 & 2.25 & 49.37 & 143 \\
Eucinostomus currani & Gerreidae & 661 & 4.00 & 53.37 & 133 \\
Orthopristis reddingi & Haemulidae & 674 & 4.08 & 57.45 & 125 \\
Haemulopsis elongatus & Haemulidae & 198 & 1.20 & 58.65 & 109
\end{tabular}

*Abu (\# Org)=Abundancia en número de organismos, Abr=Abundancia relativa, Ara=Abundancia relativa acumulada, Pun $(\mathrm{IVB})=$ Puntos con el índice de valor biológico.

scituliceps Jordan \& Gilbert, 1882, Urobatis halleri Cooper, 1863 y Diplectrum pacificum Meek \& Hildebrand, 1925 (Fig. 2 A-C). Otras especies como Haemulopsis nitidus Steindachner, 1869, Eucinostomus gracilis Gill, 1862 y Balistes polylepis Steindachner, 1876 fueron muy frecuentes en la costa Este del Golfo de California, sin embargo no se encontraron en la costa Oeste del mismo (Fig. 2 E, G y H). Por otra parte, Porichthys analis Hubbs \& Schultz, 1939 fue encontrado frecuentemente hacia el Norte y Centro del Golfo (Fig 2. K), sin embargo su presencia fue menor en la parte Sur, contrario a lo observado para Eucinostomus entomelas Zahuaranec, 1980, la cual fue muy frecuente en el sur y centro mientras que fue escasa al norte del Golfo (Fig. 2 I).

Con respecto a la distribución batimétrica de estas 15 especies, en general fueron capturadas entre los 8 y $67 \mathrm{~m}$ de profundidad (Fig. 2 A-O) y se pueden observar 3 grupos en cuanto a esto: el primero conformado por las especies U. halleri, H. nitidus, Eucinostomus argenteus Baird \& Girard, 1855, E. entomelas, Paralabrax maculatofasciatus Steindachner, 1868, Haemulopsis elongatus Steindachner, 1879 y Orthopristis reddingi Jordan \& Richardson, 1895, cuya abundancia (en número de organismos) fue mayor entre 8 y $26 \mathrm{~m}$ de profundidad (Fig. $2 \mathrm{~A}, \mathrm{D}, \mathrm{E}, \mathrm{I}, \mathrm{J}, \mathrm{N}$ y O); el segundo grupo lo conformaron $S$. scituliceps, D. pacificum, Pomadasys panamensis Steindachner, 1875, $P$. analis y Micropogonias megalops Gilbert, 1890, cuyas abundancias se concentran entre 27 y $67 \mathrm{~m}$ de profundidad (Fig. $2 \mathrm{~B}, \mathrm{C}, \mathrm{F}, \mathrm{K}$ y L) y el último grupo con abundancias más o menos constantes entre 9 y $46 \mathrm{~m}$ de profundidad, conformado por E. gracilis, 


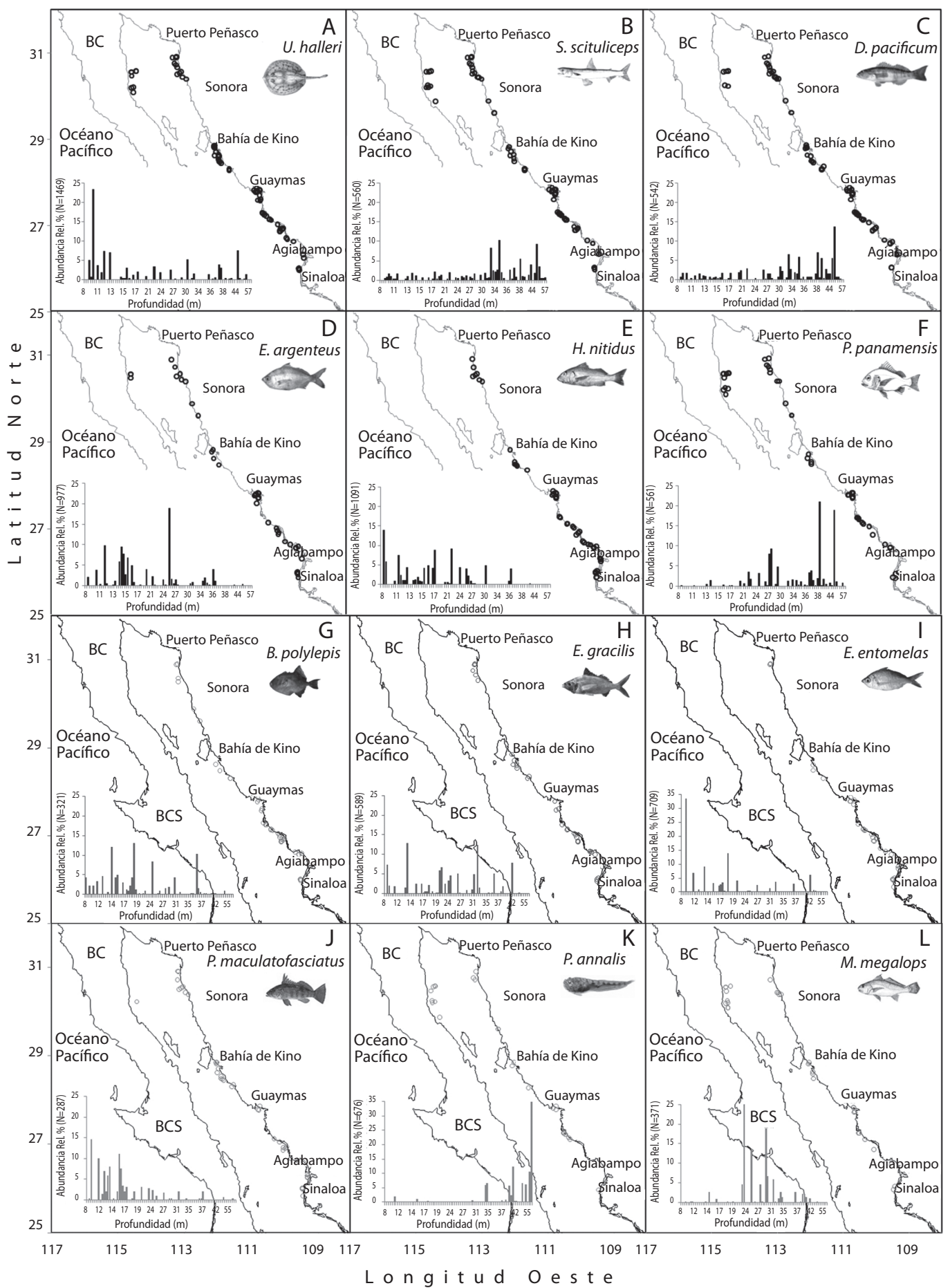

Fig. 2. Áreas de distribución latitudinal y batimétrica de las 15 especies (A-O) de mayor valor biológico en la fauna acompañante del camarón durante 2002-2005 y 2007 en el Golfo de California.

Fig. 2. Latitudinal and bathymetric distribution areas of the 15 species (A-O) with the higher biological value index in the shrimp bycatch in the Gulf of California during 2002-2005 and 2007. 


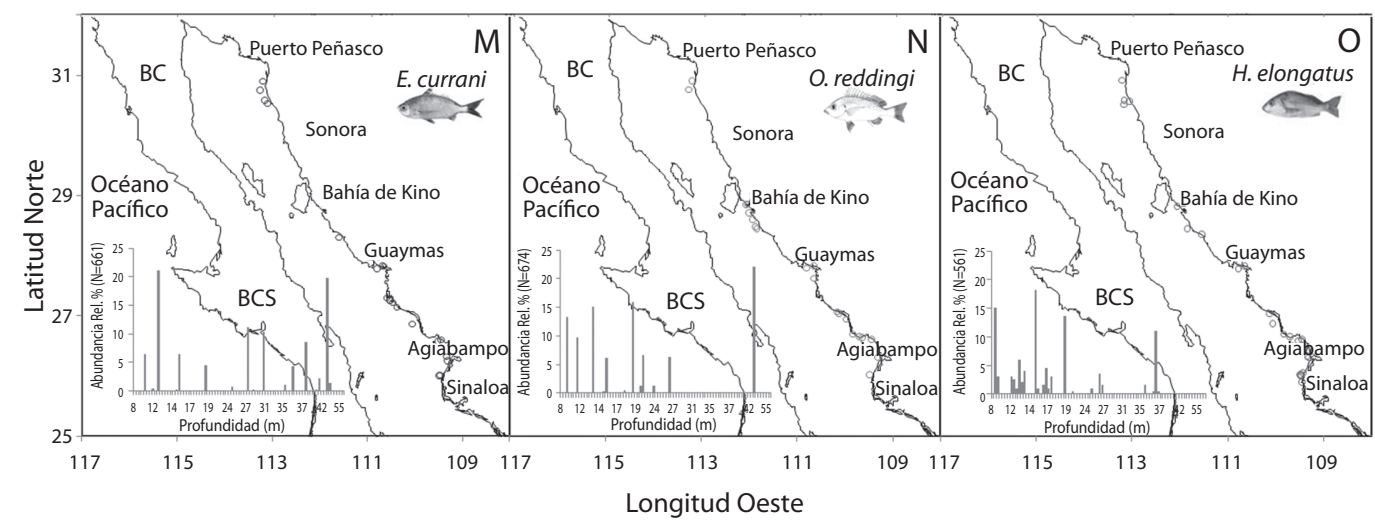

Fig. 2. (Continuación) Áreas de distribución latitudinal y batimétrica de las 15 especies (A-O) de mayor valor biológico en la fauna acompañante del camarón durante 2002-2005 y 2007 en el Golfo de California.

Fig. 2. (Continued) Latitudinal and bathymetric distribution areas of the 15 species (A-O) with the higher biological value index in the shrimp bycatch in the Gulf of California during 2002-2005 and 2007.

Eucinostomus currani Zahuaranec, 1980 y B. polylepis (Fig. $2 \mathrm{G}, \mathrm{H}$ y M).

De la fauna acompañante obtenidas en los cruceros realizados a profundidades mayores a $90 \mathrm{~m}$, sólo se encontraron 6 de las 15 especies con el IVB mas alto obtenidas en los cruceros de veda (S. scituliceps, D. pacificum, P. analis, M. megalops, P. maculatofasciatus y B. polylepis). De estas 6 especies se analizaron datos de 988 organismos y sólo dos especies fueron abundantes y frecuentes en los muestreos: $S$. scituliceps y $P$. analis, las otras cuatro aparecieron sólo en un lance.

Al combinar ambas fuentes de información para estas dos especies, se tuvo una mejor perspectiva de su distribución latitudinal y batimétrica (Fig. 3 A-B). Porichthys analis presentó una distribución centrada en la parte norte y centro del Golfo de California, con muy poca aparición hacia el sur. En cuanto a su distribución batimétrica esta especie se distribuye desde 10 a $334 \mathrm{~m}$ de profundidad y presenta las mayores abundancias entre 40 y $274 \mathrm{~m}$ (Fig. 3 A). Synodus scituliceps presentó una amplia distribución en todo el Golfo de California. En cuanto a su distribución batimétrica esta especie se distribuye desde los 9 a $280 \mathrm{~m}$ de profundidad y presenta las mayores abundancias entre los 33 y $111 \mathrm{~m}$ (Fig. 3 B).
El análisis de correspondencia mostro cierta asociación de algunas de las especies más abundantes y frecuentes en la fauna de acompañamiento del camarón con ciertas áreas del Golfo de California (Fig. 4).

\section{DISCUSIÓN}

La selección de especies representativas de una comunidad en base a datos puntuales de abundancia no resulta del todo representativa, requiriéndose de herramientas que permitan esa selección integrando cambios temporales en las dominancias de las principales especies. Una forma sencilla de realizar la descripción de la estructura por especie se basa en la aplicación de rangos y puntajes y es usada ampliamente en estudios de ecología marina, para ordenar la importancia de cada una de las especies y los sitios de muestreo de acuerdo a variables ambientales. El índice de valor biológico (IVB) propuesto por Sanders (1960) es uno de los más utilizados en este tipo de estudios ya que trabaja balanceando dos atributos numéricamente importantes: la abundancia y la constancia espacio-temporal (Sanders 1960, citado por Loya \& Escofet 1990). Para este trabajo, donde se aplicó este índice para determinar 


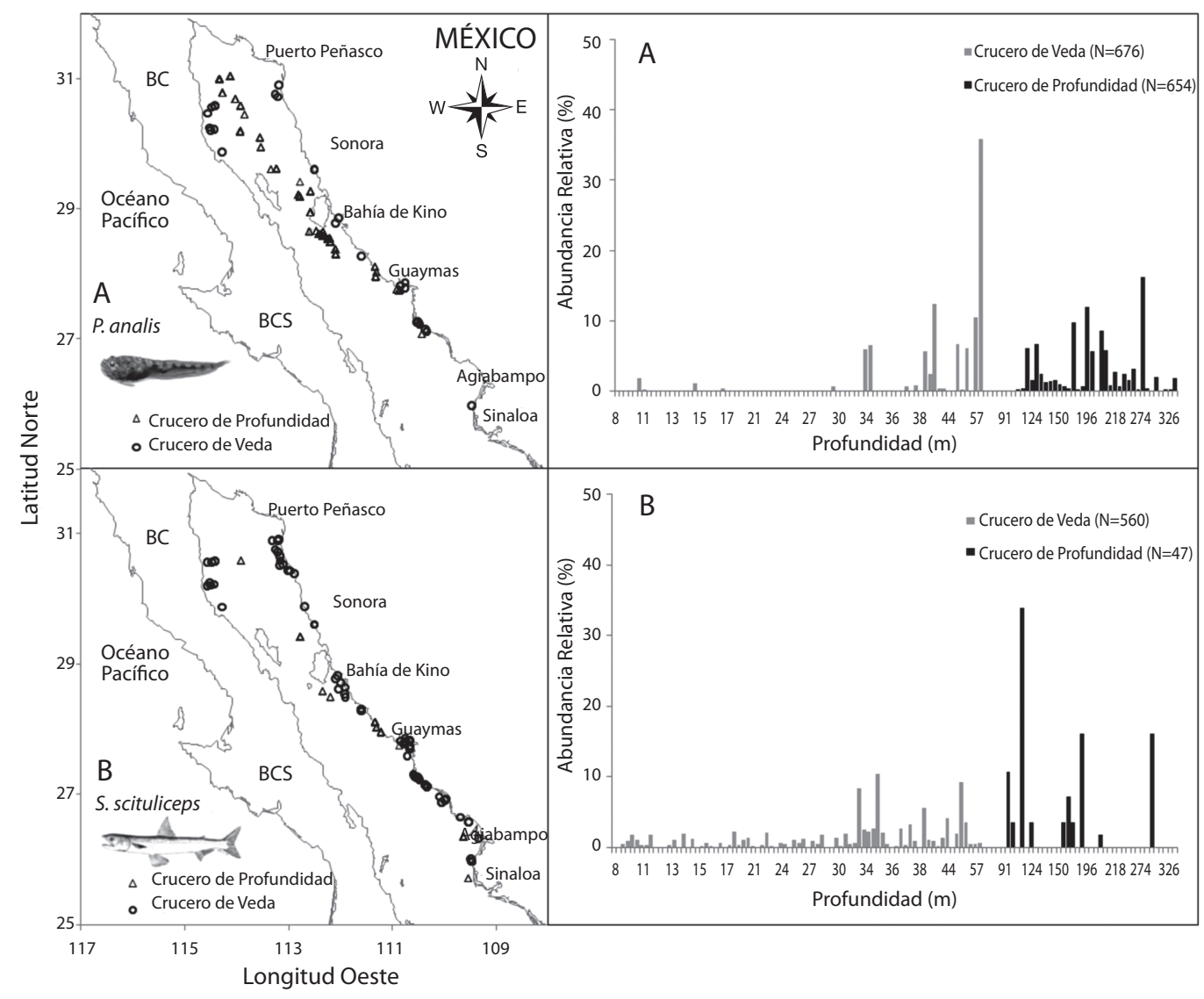

Fig. 3. Distribución latitudinal y batimétrica combinando información de los cruceros de veda y profundidad para: (A) $P$. analis y (B) S. scituliceps en el Golfo de California.

Fig. 3. Latitudinal and vertical distribution of: (A) P. analis and (B) S. scituliceps in the Gulf of California joining data collected from research cruises in the closed shrimp season and data from research cruises made at more than $90 \mathrm{~m}$ deep.

las especies de mayor importancia biológica dentro de la fauna acompañante del camarón, fue de gran ayuda ya que balanceó la constancia espacio-temporal de la abundancia de las especies y de este modo evitó la ordenación de éstas en base a datos puntuales dominantes pero poco representativos, lo cual se observó en algunas especies que fueron muy abundantes sólo en algunos lances, pero que al final fueron muy poco frecuentes en su aparición en todos los cruceros.

Las 15 especies con el IVB mas alto determinadas en el presente trabajo, se encuentran dentro de las 20 especies principales (en cuanto a frecuencia y abundancia) reportadas por Pérez-Mellado \& Finley (1985) para la misma zona, desde septiembre de 1978 a marzo de 1979. De igual forma, coinciden casi totalmente con las 15 especies más frecuentes y abundantes en el estudio realizado por LópezMartínez et al. (2007b), donde muestrearon la fauna asociada al camarón tanto de la operación de la flota camaronera como en cruceros realizados en época de veda, a lo largo de todo el Golfo de California.

Por otra parte, coinciden (en cuanto a su abundancia) con varias de las principales especies reportadas por Madrid-Vera et al. (2007), 


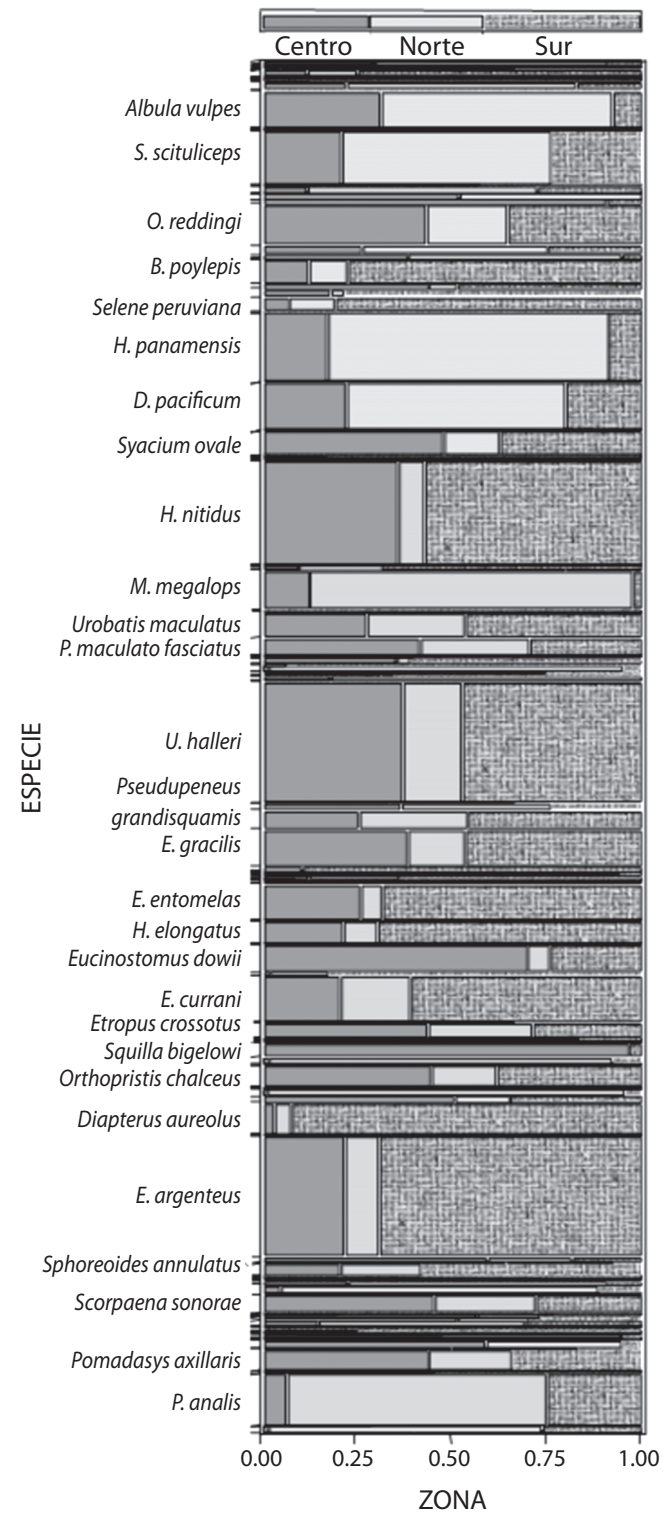

Fig. 4. Ordenación y asociación de las especies más abundantes y frecuentes de la fauna acompañante del camarón con determinada zona del Golfo de California, durante la temporada de veda de 2002-2005 y 2007.

Fig. 4. Ordination and association of the most abundant and frequent species in the shrimp bycatch with determinate zone from the Gulf of California, during shrimp closed season 2002-2005 and 2007. quienes realizaron muestreos de la fauna asociada al camarón en cruceros en época de veda en el Golfo de California.

Lo anterior refleja que existe una gran similitud en cuanto a las especies más abundantes y frecuentes acompañantes del camarón obtenidas tanto por muestreos realizados en cruceros de veda (julio y agosto) como provenientes de la pesca comercial (septiembreabril). Por lo que las especies más abundantes y frecuentes a través del IVB obtenidas en este trabajo, nos dan una noción fidedigna de las especies que son mayormente capturadas por la flota camaronera en el Golfo de California. Sin embargo, se deben tomar en cuenta las variaciones en la composición de las especies durante todo el año.

Catorce de las 15 especies de mayor importancia biológica, presentan una distribución amplia, ya que son endémicas del Pacífico Este y regionalmente endémicas y residentes del Pacífico Oriental Tropical (Fischer et al. 1995, Robertson \& Allen 2002, Froese \& Pauly 2009). Esto concuerda con lo reportado por Castro-Aguirre et al. (1995), quienes mencionan que la ictiofauna del Golfo de California pertenece en su mayoría a la región del Pacífico Oriental Tropical, que debido a su diversidad topográfica, batimétrica, climática, etc., proporciona un gran número de hábitats para los peces y otros organismos. Por otra parte, sólo E. argenteus presenta una distribución global más amplia, distribuyéndose tanto en el Pacifico Oriental Tropical como en el Atlántico Oeste (Froese \& Pauly 2009), concordando esto también con lo descrito por Walker (1960), citado por Castro-Aguirre et al. (1995), quien menciona que $73 \%$ del total de las especies ícticas del Golfo de California está constituida por elementos de la región Panámica, 10\% de formas ártico-boreales (incluyendo especies compartidas con la ictiofauna Californiana, Oregoniana y Canadiense) y $17 \%$ endémicas.

Regionalmente, las especies $U$. halleri, $S$. scituliceps, $D$. pacificum, $H$. nitidus, E. agenteus, $P$. panamensis, E. gracilis, $P$. analis, $M$. megalops, E. currani, O. reddingi presentan una distribución a lo largo de todo el Golfo de 
California. La amplia distribución latitudinal de éstas puede ser debida a que son especies que se han ido adaptando a la gran variedad de hábitats y condiciones ambientales del Golfo de California (López-Martínez et al. 2010).

Por otra parte, P. maculatofasciatus y $B$. polylepis se distribuyen en casi todo el Golfo a excepción del sur de Sinaloa y Nayarit. Según Robertson \& Allen (2002), la especie E. entomelas no se encuentra distribuida en la parte alta del Golfo de California y sí desde los tres cuartos inferiores del Golfo hasta Perú, coincidiendo con los resultados aquí reportados.

La distribución latitudinal más acotada para estas especies puede ser un claro ejemplo que algunos peces pueden tener barreras térmicas y de tipo topográficas (batimétrica y sedimentaria) que impiden su distribución más allá de ciertos límites, lo cual ha sido muchas veces citado en estudios zoogeográficos para el Golfo de California. Sin embargo, dichas barreras no tienen el mismo efecto para todos los elementos de la ictiofauna del Golfo, por lo que a estas barreras se les considera más bien como "filtros faunísticos" (Castro-Aguirre et al. 1995).

Por ejemplo, barreras topográficas constituidas por grandes extensiones de costa arenosa, que se caracteriza por varios sistemas estuario-lagunares entre el sur de Guaymas y Mazatlán, han restringido el establecimiento en el Golfo de ciertas especies típicas de ambientes rocosos o coralinos. Por otra parte, la costa del lado Baja Californiano que es en su mayoría rocosa, con acantilados y accidentada en su desarrollo, impide el establecimiento de especies típicas de áreas arenosas o fangosas. Se ha planteado que la diversidad de especies varía según los tipos de sedimento, ya que de ello depende la disponibilidad de alimento determinante en la abundancia de las especies (Gage \& Tyler 1991) y se ha propuesto que existe una clara diferencia entre la fauna de la plataforma continental y del talud continental (Powell et al. 2003) debido principalmente a factores como el nivel de luz, sustrato y sedimentos así como temperatura, oxígeno disuelto y salinidad (Fujita et al. 1995).
Los gradientes térmicos en el Golfo de California, son uno de los filtros faunísticos que determinan en gran medida la distribución de los peces y otros organismos, donde la temperatura juega un papel como factor limitante en la distribución y abundancia de varias especies, sobre todo aquellas que viven en ambientes arenosos o lodosos (la mayoría de las aquí estudiadas); además que estas especies puedan poseer un fuerte componente estuario-lagunar en alguna fase de su ciclo de vida, lo cual también sería una barrera para su distribución (Castro-Aguirre et al. 1995).

De acuerdo con Robertson \& Allen (2002), $H$. elongatus se distribuye desde la boca del Golfo de California a Perú, pero no dentro del mismo, mientras que en el presente trabajo se reporta la ampliación del rango de distribución de esta especie, la que fue encontrada en la mayor parte de los lances muestreados a lo largo del Golfo.

La mayoría de las especies analizadas son demersales asociadas al fondo o cercanas al fondo marino, con una amplia distribución batimétrica (9 a $67 \mathrm{~m}$ de profundidad) y en 2 de estas especies ( $S$. scituliceps y $P$. analis) hasta los $325 \mathrm{~m}$. Estos rangos de distribución batimétrica corresponden a los reportados para estas especies (Froese \& Pauly 2009, Fischer et al. 1995, Robertson \& Allen 2002). Al igual que la distribución latitudinal, la distribución batimétrica de las especies puede estar limitada por barreras térmicas y de presión así como de tipo topográfico presentes en el Golfo de California. La distribución batimétrica (9-67m) de las especies aquí estudiadas, puede ser debida a que en la parte Oriental del Golfo de California existen grandes extensiones costeras arenoso-fangosas que son áreas muy productivas y soportan una gran diversidad de especies ícticas y otros organismos, áreas donde estas especies son muy comunes (López-Martínez et al. 2010).

Si bien el analizar información de las capturas incidentales con muestreos independientes de la pesquería comercial de camarón nos puede dar una visión más amplia en cuanto a la distribución de estas especies (ya que no 
estaría sesgada por el muestrear solo áreas donde se capturan las especies de camarón "caladeros"), se deben tomar en cuenta los posibles cambios temporales en la distribución batimétrica, ya que algunas de estas especies pueden tener migraciones con fines reproductivos (hacia aguas más profundas, o hacia aguas más someras).

En particular para S. scituliceps y P. analis, el amplio rango de distribución batimétrica que presentan podría tener repercusiones en su supervivencia y en el potencial efecto que ejerce sobre ellas la pesca de arrastre que se efectúa en el Golfo de California. Si se considera que de acuerdo a Morales-Azpeitia (com. pers.), la mayor parte $(73 \%)$ de los arrastres camaroneros en el Golfo de California se efectúa de los 8 a los 35m; y que en el presente trabajo se encontró que ambas especies presentan abundancias relativas significativas más allá de los $35 \mathrm{~m}$ (al igual que otras especies con su distribución cargada a estas profundidades), esto es, podría haber una fracción importante de la biomasa de ambas especies que están en áreas poco o no impactadas por los arrastres. Por lo que los efectos que la pesca de arrastre puede tener en ambas especies pudieran ser menores a los que inicialmente se piensa.

Por lo anterior, es necesario efectuar estudios posteriores para determinar las abundancias (relativas y si es posible absolutas) por estrato de profundidad para cada una de estas especies y evaluar el escape proporcional (\% de biomasa no capturada por la flota) que se pudieran tener en esta pesquería multiespecífica. De igual forma, es necesario evaluar las tallas a las que se presentan estas especies por estrato de profundidad, para determinar cuáles son las más vulnerables a la pesca.

\section{AGRADECIMIENTOS}

La información con la que se realizó este trabajo fue financiada por los proyectos SAGARPA-CONACYT 2003-002-089 y 2003002-024 y el proyecto EP1.1 del CIBNOR. Se agradece al Laboratorio de Pesquerías del CIBNOR Guaymas, en particular a Eloisa Herrera
Valdivia. Se agradece al Instituto Nacional de Pesca y a la Comisión Nacional de Pesca por el Permiso de Pesca de Fomento No. DGOPA.02226.110407-0937. El primer autor es becario CONACYT (219000). JEVH recibió apoyo de CONACYT Proyecto FLUCARGOC II (84944). Joaquín Magaña corrigió el resumen en inglés.

\section{RESUMEN}

El Golfo de California es una de las regiones más mega diversas en el mundo, del cual poca información pesquera está disponible. Aquí se presenta la distribución latitudinal y batimétrica de las especies más abundantes y frecuentes en la fauna acompañante del camarón del Golfo de California. Las muestras fueron obtenidas de un total de 111 lances provenientes de siete cruceros en época de veda del camarón (2002-2005-2007) y prospecciones realizadas a profundidades mayores a $10 \mathrm{~s} 90 \mathrm{~m}$. Debido al gran número de especies en esta fauna asociada al camarón, se eligieron las que presentaron el índice de valor biológico (IVB) más alto. Las 15 especies con IVB más alto representaron el $60 \%$ de la abundancia total. Un total de 16508 organismos fueron analizados representando 243 especies entre peces, crustáceos, moluscos y equinodermos. El grupo de los peces fue el más abundante, siendo las más abundantes y frecuentes: Urobatis halleri, Synodus scituliceps, Diplectrum pacificum, Haemulopsis nitidus y Eucinostomus argenteus. Se observó una amplia distribución latitudinal de estas especies a lo largo del área de estudio y una distribución batimétrica desde los 9 hasta los $67 \mathrm{~m}$ de profundidad. Dos de ellas se distribuyeron hasta los $325 \mathrm{~m}$. Debido a esta amplia distribución batimétrica, se hace necesario determinar las abundancias totales y tallas por estrato de profundidad para cada especie, ya que a profundidades mayores de $25 \mathrm{~m}$ la captura de éstas puede ser más baja y pueden servir como áreas de repoblación natural para las profundidades donde son capturadas principalmente por la flota camaronera comercial.

Palabras clave: índice de valor biológico, arrastre camaronero, temporada de veda camaronera, fauna acompañante, peces.

\section{REFERENCIAS}

Bojorquez, L.F. 1998. Bycatch utilization in Mexico. Report and Proceedings on the FAO/DFID Expert Consultation on Bycatch Utilization in Tropical Fisheries, Bejing, September 1998. FAO, Rome.

Castro-Aguirre, J.L., E.F. Balart-Páez \& J. Arvizu-Martínez. 1995. Contribución al conocimiento del origen y 
distribución de la ictiofauna del Golfo de California, México. Hidrobiológica. 5: 57-58.

Davis, M.W. 2002. Key principles for understanding fish bycatch discard mortality. Can. J. Fish. Aquat. Sci. 59: 1834-1843.

Eschmeyer, W.N., E.S. Herald \& H Hammann. 1983. A field guide to the Pacific coast fishes of North America. Hougthon Mifflin Harcourt, Boston.

Fischer, W., F. Krupp, W. Schneider, C. Sommer, K.E. Carpenter \& V.H. Niem. 1995. Guía FAO para la identificación de especies para los fines de pesca. Pacífico Centro-Oriental. FAO, Roma.

Froese, R. \& D. Pauly. 2009. Fish Base. A Global Information System on Fishes. (Consultado: Octubre 2010, www.fishbase.org).

Fujita, T., T. Inada \& Y. Ishito. 1995. Depth gradient structure of the demersal fish community of the continental shelf and upper slope off Sendai Bay, Japan. Mar. Ecol. Prog. Ser. 118: 13-23.

Gage, J.D. \& P.A. Tyler. 1991. Deep sea biology: A natural history of organisms at the deep sea floor. Cambridge, England.

Jordan, D.S. \& B.W. Evermann. 1896-1900. The fishes of North and Middle America. Part. I (1896), II (1898), III (1898) \& IV (1900). Bulletin of the United States Natural History Museum. 47: 1-3313.

Kanyerere, G.Z. 2001. Spatial and Temporal Distribution of Some Commercially Important Fish Species in the Southeast and Southwest Arms of Lake Malawi: A Geostatistical Analysis. Fish. Bull. 43: 1-30.

Kelleher, K. 2005. Discarding in the world's fisheries: an update. FAO Fisheries Technical Paper 470. FAO, Roma.

López-Martínez, J., S. Hernández-Vázquez, C.H. RábagoQuiroz, E. Herrera-Valdivia \& R. Morales-Azpeitia. 2007a. Efectos ecológicos de la pesca de arrastre de camarón en el Golfo de California. Estado del arte del desarrollo Tecnológico de las Artes de Pesca, p. 14-47. In CEDRSSA (eds.). La situación del sector pesquero en México. CEDRSSA y Cámara de diputados LX Legislatura, México, D.F.

López-Martínez, J., S. Hernández-Vázquez, N. HernándezSaavedra, J. Rodríguez-Romero, E. Balart-Páez, E. Herrera-Valdivia, R. Morales-Azpeitia, C. RábagoQuiroz, A. Siaruqui-Quijano, J. Padilla-Serrato \& A. Salazar-Ayala. 2007b. Dinámica poblacional de especies presentes en la captura incidental de la pesquería de camarón del Golfo de California. Producto 5 del Informe final del proyecto SAGARPA-CONACYT, Evaluación del impacto que ocasionan las redes de arrastre para camarón y escama demersal en los fondos marinos del Golfo de California. CIBNOR, Guaymas, Sonora, México.

López-Martínez J., E. Herrera-Valdivia, J. RodríguezRomero \& S. Hernández-Vázquez. 2010. Composición taxonómica de peces integrantes de la fauna de acompañamiento de la pesca industrial de camarón del Golfo de California, México. Biol. Trop. 58: 925-942.

López-Martínez, J., E. Morales-Bojórques, F. ParedesMallón, D. Lluch-Belda \& C. Cervantes. 2001. La pesquería de camarón de altamar en Sonora, p. 301312. In D. Lluch-Belda, J. Elorduy-Garay, S. LluchCota \& G. Ponce-Díaz (eds.). Centros de actividad biológica del Pacífico Mexicano. CIBNOR/CONACYT, La Paz, México.

Loya-Salinas, D.H. \& A. Escofet. 1990. Aportaciones al cálculo del Índice de Valor Biológico (Sanders, 1960). Ciencias Marinas. 10: 97-115.

Madrid-Vera, J., F. Amezcua-Linares \& E. Morales-Bojórques. 2007. An assessment approach to estimate biomass of fish communities from bycatch data in a tropical shrimp-trawl fishery. Fish. Res. 83: 81-89.

Meek, S.E \& S.F. Hildebrand. 1923-1928. The marine fishes of Panama. Publications of the Field Museum of Natural History Zoological Series 15: 1-1045.

Miller, D.J. \& R.N. Lea. 1976. Guide to the coastal marine fishes of California. California Department of Fish and Game. Fish. Bull. 157: 1-249.

Pérez-Mellado, J. \& L.T. Findley. 1985. Evaluación de la ictiofauna acompañante del camarón en las costas de Sonora y norte de Sinaloa, México, p. 149-200. In A. Yáñez-Arancibia (eds.). Recursos pesqueros potenciales de México: la pesca acompañante del camarón. Ciencias del Mar y Limnología, UNAM, México, D.F.

Pope, J.G., D.S. MacDonald, J.D. Reynolds \& S. Jennings. 2000. Gauging the impact of fishing mortality on non-target species. ICES J. Mar. Sci. 57: 689-696.

Powell, S.M., R.L. Haendrich \& J.D. McEachran. 2003. The deep demersal fish fauna of the Northern Gulf of Mexico. J. Northwest Atl. Fish. Sci. 31: 19-33.

Rábago-Quiroz, C.H., J. López-Martínez, E. Herrera-Valdivia, M.O. Nevárez-Martínez \& J. Rodríguez-Romero. 
2008. Population dynamics and spatial distribution of flatfish species in shrimp trawl bycatch in the Gulf of California. Hidrobiológica 18: 177-188.

Robertson, D.R. \& G.R. Allen. 2002. Shorefish of the tropical eastern Pacific: An information system. Smithsonian Tropical Research institute, Balboa, Panama. (Consultado: Octubre 2010, www.stri.si.edu/sftep)
Rueda, M. \& O. Defeo. 2001. Survey abundance indices in a tropical estuarine lagoon and their management implications: a spatially-explicit approach. ICES J. Mar. Sci. 58: 1219-1231.

Stobutzki, I., P. Jones \& M. Miller. 2003. A comparison of fish bycatch communities between areas open and closed to prawn trawling in an Australian tropical fishery. ICES J. Mar. Sci. 60: 951-966. 